\title{
Multidrug Resistant Probiotics as an Alternative to Antibiotic Probiotic therapy
}

\author{
Dhanashree Lokesh', Kammara Rajagopal ${ }^{1,2}$, Jae Ho Shin ${ }^{2 *}$ \\ 'Department of Protein Chemistry and Technology, CSIR-CFTRI, Mysore, India \\ ${ }^{2}$ School of Applied Biosciences, Kyungpook National University, Daegu, Korea
}

\section{Article Info}

\section{Article Notes}

Received: September 30, 2019

Accepted: November 20, 2019

\section{*Correspondence:}

Dr. Jae-Ho Shin, School of Applied Biosciences,

Kyungpook National University, Daegu, Korea.

(c) 2019 Shin JH. This article is distributed under the terms of the Creative Commons Attribution 4.0 International License.
Abstract

Drug-resistance is a major problem globally, the number of drug-resistant bacteria has increased substantially through horizontal gene transfer. Even Mycobacterium tuberculosis are reported to have acquired antitubercular drug-resistance and named as MDR Mtb. The acquisition of immunity has not given up, here; it is needed to be a continuous procedure. Further causing the microbial adapting to a very high and larger number of drugs recognized as extreme drug and total drug-resistance. The mechanistic aspects of MDR Mtb are well understood. Nevertheless, this is not the case with Probiotic microbes such as Bifidobacterium adolescentis. Herein, we report the mechanistic aspects of antitubercular drug-resistance in this organism for the first time. This review discusses the report by a mutation that confers multi drug-resistance in Bifidobacteria.

\section{Introduction}

Penicillin was introduced for the first time in $1928^{1}$. Afterward, in the 1940s, bacterial resistance against penicillin developed, which made headlines. Later, to overcome this resistance various antibiotics came into the market ${ }^{2}$. At late times, the world is facing a crisis in terms of antibiotic resistance ${ }^{3}$. Bacterial resistance is inevitable and its evolution is uncertain ${ }^{4}$. Pharmaceutical companies initially gave up investing in designing new antibiotics; this was accompanied by a decrease in the use of antibiotics, and finally the minimization of new drug introduction a continuous process ${ }^{5}$. The best means to circumvent antimicrobial resistance, the widespread and indiscriminate use of antibiotics is to use an alternative in the form of probiotics. One of the most important characteristics of probiotics is the synthesis and secretion of antimicrobial proteins/ peptides. Probiotics are simply not very effective in providing good health effects exhibiting health benefits, but likewise do not introduce antimicrobial resistance in the target organism, as antibiotics do. Hence, to address the pressing need, the only effect is the economic consumption of probiotics as an alternative to antibiotics to treat few diseases caused by pathogens ${ }^{6}$. Probiotics, defined as live microbial consortia, have the power to offer secure health and also replenish gut flora ${ }^{7}$.

Globally, many laws and ordinances have been implemented for the ethical use of microbes for therapy and good health. The most important and efficacious of these are the European laws. According to European standards, microbes should undergo Qualified Presumption of Safety (QPS) tests before human use can be considered ${ }^{8}$. Lactobacillus and Bifidobacteria are two major 
inhabitants of the host and a few species are used as starter cultures in many food industries ${ }^{9}$. In that respect are various commercially available probiotics with Generally Recognized As Safe (GRAS) status recommended for human consumption that are described to cause antibiotic-resistant genes ${ }^{10}$. These are believed safe for human consumption as they do not cause any disease, and more significantly, on that point is a low risk/negligible risk of shifting the resistance gene present in genomic DNA $^{11}$. To date, there are no reports of horizontal transfer of antibiotic resistance genes between probiotics and pathogenic microbes or vice versa.

Globally, the most common and infective/pathogenic microbes are Salmonella, Listeria, Vibrio spp, and so on. The other most dreaded microbes that cause chronic disease is Mycobacterium tuberculosis. Until lately, this was seen as a disease of the impoverished as it was mentioned frequently in epidemics only in developing nations. However, the causative agent of tuberculosis recognized and found in developed and advanced countries is more virulent. The World Health Organization (WHO) reports suggest that one-third of the population gets infected every year by Mycobacterium tuberculosis ${ }^{12}$, and most recover due to effective resistance. Only about 5-10\% develop active tuberculosis ${ }^{13}$. Antimicrobial resistance to Mycobacterium tuberculosis has led to the creation of multidrug resistant (MDR), extensive drug resistant (XDR), and total drug resistant (TDR) forms of tuberculosis. According to WHO guidelines, drug-sensitive tuberculosis can be treated by administering a combination of four firstline antibiotics, namely Rifampicin, Isoniazid, Ethambutol, and Pyrazinamide for two months and subsequently, rifampicin and isoniazid for at least four months on a daily basis $^{14,15,16}$. Out of the four, rifampicin alone shows broadspectrum activity ${ }^{17}$, whereas the other three specifically target the Mycobacterial spp ${ }^{18-20}$.

Drug-resistant strains of Mycobacterium tuberculosis have originated mainly due to intermittentand discontinuous use of prescribed drugs during therapy. At the molecular level, anti-tubercular drug resistance is primarily due to modification of the rpo $\beta$ gene. The modified RNA polymerase $\beta$ subunit (rpo $\beta$ ) (a housekeeping gene) loses its shape so that it no longer has the capacity to bind to make a complete protein in order to function. Thus, in one case the change is negatively affected, subsequently it stops participating in protein translation/transcription, ultimately creating drug resistant Mycobacterium tuberculosis. Studies show that mutations in the rpo $\beta$ gene lead to rifampicin resistance and also affect the glutamine metabolism ${ }^{21}$.

In present day circumstances, many organisms similar to Mycobacterium tuberculosis are acquiring resistance to firstline anti-tubercular drugs in a similar fashion. To mention a few, organisms, Escherichia coli, Staphylococcus aureus,
Haemophilus influenzae, Neisseria meningitides, Streptococcus pneumoniae, Rhodococcus equi, and Bifidobacteria are intrinsically resistant to rifampicin ${ }^{22,23}$. A few others, including Pseudomonas fluorescence, Enterobacteriaceae, Treponema spp, Borrelia, Leptospira, Mycoplasma, Urea-plasma, and Spiroplasma species shown resistance due to membrane impermeability, plasmid-mediated efflux systems, influx/ efflux prophylaxis, and refractory ribonucleic acid polymerase (RNAP) ${ }^{24-27}$. Nocardia spp. Show resistance to rifampicin by inactivating the drug by glycosylation, ribosylation, phosphorylation, and de-colorization ${ }^{28-30}$. What is alarming is that recently a few of the GRAS organisms, such as Bifidobacterium adolescentis have also been observed to be multidrug resistant.

A study on controlling tuberculosis pathogenicity using gut microbiota revealed that altered gut flora due to antibiotic perturbation increases the mycobacterial burden in the lungs and also leads to dissemination to other organs, such as spleen and liver ${ }^{31}$. Antibiotics are the cornerstone of innovative health measures; intake of different classes of antibiotics, which are explicitly or broadly designed to kill bacteria leads to depletion of commensals, including gut bacteria ${ }^{32}$. In such cases, development of multidrugresistant gut microbes becomes a critical issue ${ }^{33}$. Therefore, it is very essential to understand the mechanistic aspects of resistance to first-line anti-tubercular drugs in probiotic microbes, specifically concerning Bifidobacterium adolescentis as a model organism. This review focuses on the studies carried out with anti-tubercular drug resistance in Bifidobacteria.

In our previous work published in Scientific Reports in 2018, we reported that probiotic organisms, such as $B$. adolescentis, $B$. animalis, and $B$. longum show resistance and also adapt to a higher concentration of rifampicin and grow luxuriantly in the presence of the other three firstline antitubercular drugs. Apart from these, there are many commercially available probiotics reported to be multidrug resistant and capable of horizontal gene transfer of pathogens, as well as other commensal present in the human gut $^{34}$. Inherited resistance in probiotics can be genetic-, plasmid-, or transposon-mediated. Getting rid of the plasmid responsible for immunity from the probiotic strains before using them as a food supplement is one of the best ways to avoid horizontal gene transfer ${ }^{35}$. Acquired resistance occurs because of cell wall modifications, active efflux systems, enzymatic intoxication, target modification, or metabolic re-arrangement ${ }^{36,37}$. For developing new treatment strategies, it is necessary to counteract the adverse effects of antibiotics on the host microbiome ${ }^{38}$.

\section{Concluding remarks}

The study concludes that even Bifidobacteria show antitubercular resistance. Being a GRAS organism, this is not 
only very alarming and dangerous, but also throws doubt over their use as probiotic microbes. There are two different ways to use these microbes for human betterment despite their anti-tubercular resistance. The most significant way may be to take out the plasmid from the organism that causes the impedance. All the same, the problem rises if it is genomic in origin.

The other strategy could be supplementing antibioticresistant probiotics for antibiotic probiotic therapy. Prior to adopting new drugs in probiotics, it is necessary to determine and record inherited, as well as spontaneous mutations. If there is a resistance gene, which is likely to be transferred, it should be removed before therapy. A combination of two or three inherently resistant probiotics for different antibiotics is also one of the best choices for treatment. Creating a database with a GRAS organism screened for antibiotic-resistance gene profiles in a single platform will be advantageous for future use.

\section{Acknowledgments}

This work was supported by the Strategic Initiative for Microbiomes in Agriculture and Food, Ministry of Agriculture, Food and Rural Affairs, Republic of Korea, grant number 918010-4. And we wish to thank the National Research Federation (NRF, Korea) for the Brain Pool Fellowship provided to KR. We would like to thank the Director CSIR-CFTRI, for providing Sabbatical to KR.

\section{References}

1. Sengupta S, Chattopadhyay MK, Grossart HP. The multifaceted role of antibiotics and antibiotic resistance in nature. Frontiers in Microbiol. 2013; 4: 47.

2. Spellberg B, Gilbert N. The future of antibiotics and Resistance: A Tribute to a career leadership by John Bartlett. 2014; 59: 71-75.

3. States U. Antibiotic resistance threats in the United States: A CDC report. 2013; 4: 23.

4. Ian MG, Abhijit MB. New antibiotic agents in the pipeline and how they can help overcome microbial resistance threats. Virulence. 2013 Feb 15; 4 (2): 185-191.

5. Ventola CL. The antibiotic resistance crisis: Part 1: Cause and threats. 2015; 40: 277-83.

6. Muñoz-Atienza E, Gómez-Sala B, Araújo C, et al. Antimicrobial activity, antibiotic susceptibility and virulence factors of Lactic acid bacteria of aquatic origin intended for use as probiotics in aquaculture. BMC Microbiol. 2013; 13: 15.

7. Verschuere L, Rombaut G, Sorgeloos P, et al. Probiotic bacteria as biological control agents in Aquaculture. Microbiol Mol Biol Rev. 2000; 64: 655-671.

8. Technical guidance-updates of the criteria used in the assessment of bacterial resistance to antibiotics of human or veterinary importance. EFSA J. 2008; 7: 14.

9. Gueimonde M, Sanchez B, de los Reyes-Gavlan, et al. Antibiotic resistance in probiotic bacteria. Frontiers in Microbiol. 2013; 4: 202.

10. Liu C, Zhang ZY, Dong K, et al. Antibiotic resistance of probiotic strains of Lactic acid bacteria isolated from marketed Foods and Drugs. Biomed Environ Sci. 2009; 22: 401-412.
11. Cannon JP, Lee TA, Bolanos JT, et al. Pathogenic relevance of Lactobacillus: A Retrospective review of over 200 cases. Eur J Clin Microbiol Infect Dis. 2005; 24: 31-40.

12. World Health Organization- Global tuberculosis report. 2016; ISBN 9789241565394.

13. Druszczynska M, Kowalewicz-kulbat M, Fol M, et al. Latent $M$. tuberculosis infection-pathogenesis, diagnosis, treatment and prevention strategies. Pol J Microbiol. 2012; 61(1): 3-10.

14. Guidelines for the treatment of tuberculosis. WHO 2010; 147.

15. EAMR- AR of and 1981. Un-define, controlled clinical trial of Five Short-course chemotherapy Regimen in Pulmonary Tuberculosis: Second report of the $4^{\text {th }}$ study. Tubercle. 1980; 61(2): 59-69.

16. World Health Organization. Global tuberculosis report. 2013. World Health Organization. https://apps.who.int/iris/ handle/10665/91355.

17. Brennan PJ, Young B. Rifampin. Tubercle. 2008; 88: 151-154. doi:10.1016/S1472-9792 (08)70024-6.

18. Brennan PJ, DBY. Ethambutol. Tubercle. 2008; 88: 102-105.

19. Brennan PJ, Young DB. Pyrazinamide. Tubercle. 2008; 88: 141-144.

20. Brennan PJ, Young DB, BDR. Isoniazid. Tubercle. 2008; 88: 112-112.

21. Morishita T, Yura T. Altered nutritional requirements associated with mutations affecting the structures of ribonucleic acid polymerase in Lactobacillus casei. J Bacteriol. 1976; 125: 416-22.

22. Goldstein BP. Resistance to rifampicin: A review. J Antibiot. 2014; 67(9): 625-30. doi: 10.1038/ja.2014.107.

23. Lokesh D, Parkesh R, Kammara R. Bifidobacterium adolescentis is intrinsically resistant to antitubercular drugs. Sci Rep. 2018; 8: 11897.

24. Louw GE, Warren RM, Gey van Pittius NC, et al. A Balancing act: Efflux/Influx in Mycobacterial Drug Resistance. Antimicrob. Agents Chemother. 2009; 53: 3181-3189.

25. Chandrasekaran S, Lalitha kumari D. Plasmid mediated rifampicin resistance in Pseudomonas fluoresence. J Med Microbiol.1998; 47: 197-200.

26. Stamm LV, Bergen HL, Shangraw KA. Natural rifampin resistance in Trepanoma spp., correlates with the presence of N531 in RpoB Rif cluster I. Antimicrob. Agents Chemother. 2001; 45: 2973-4.

27. Kim H, Kim SH, Ying YH, et al. Mechanism of natural rifampin resistance of Streptomyces spp. Syst Appl Microbiol. 2005; 28: 398-404.

28. Andersen SJ, Quan S, Gowan B, et al. Mono-oxygenase-like sequence of a Rhodococcus equi gene conferring increased resistance to rifampin by inactivating this antibiotic. Antimicrob. Agents Chemother.1997; 41: 218-21.

29. Quan S, Venter H, Dabbs ER. Ribosylative inactivation of rifampin by Mycobacterium smegmatis is a principal contributor to its low susceptibility to this antibiotic. Antimicrob. Agents Chemother.1997; 41: $2456-60$

30. Tupin $A$, Gualtieri $M$, Roquet-Banères $F$, et al. Resistance to rifampicin: At the crossroads between ecological, genomic and medical concerns. Int J Antimicrob Agents. 2001; 35: 519-523.

31. Nargis Khan, Aurobind Vidyarthi, Sajid Nadeem, et al. Alteration in the Gut Microbiota Provokes Susceptibility to Tuberculosis. Frontiers in Immunology. 2016; 7: 529.

32. Langdon A, Crook N, Dantas G. The effects of antibiotics on the microbiome throughout development and alternative approaches for therapeutic modulation. Genome Med. 2016; 8: 39.

33. Jose NM, Bunt CR, Hussain MA. Implications of Antibiotic Resistance in Probiotics. Food Rev Int. 2015; 31: 52-62. 
34. Sharma P, Tomar SK, Goswami P, et al. Antibiotic resistance among commercially available probiotics. Food Res Int. 2014; 57: 176195.

35. Aloysius Wong, Davey Yueh Saint Ngu, Lydia Annabel Dan, et al. Detection of antibiotic resistance in probiotics of dietary supplements. 2015; doi:10.1186/s12937-015-0084-2
36. Holzbauer S, Chiller T. Antimicrobial Resistance in Bacteria of Animal Origin. Emerg Infect Dis. 2006; 12: 1180-1180.

37. Courvalin P. Antibiotic resistance: the pros and cons of probiotics. Dig Liver Dis. 2006; 38: S261-S265.

38. Wood MR, Yu EA, Mehta S. The Human microbiome in the Fight Against Tuberculosis. Am J Trop Med Hyg. 2017; 96: 1274-1284. 